\title{
componentes y elementos de catálogo: una vía de racionalización del proceso constructivo
}

Julián Salas, Ingeniero Industrial

\section{sinopsis}

Se analiza el concepto de componente constructivo, su repercusión económica, mediante una división de la vivienda en elementos funcionales, asi como las distintas utilizaciones que pueden hacerse de los componentes en construcción.

El tema de los elementos de catálogo se plantea buscando sus origenes en los catálogos de elementos de fundición del XIX estableciendo, más adelante, una división entre elementos especificos y autónomos. Finalmente el trabajo plantea lo que pudieran ser condiciones minimas para abordar unas reglas de juego autóctones minimas para abordar unas reglas de juego autoctonas que facilitasen mentos de catálogo aqui y ahora.

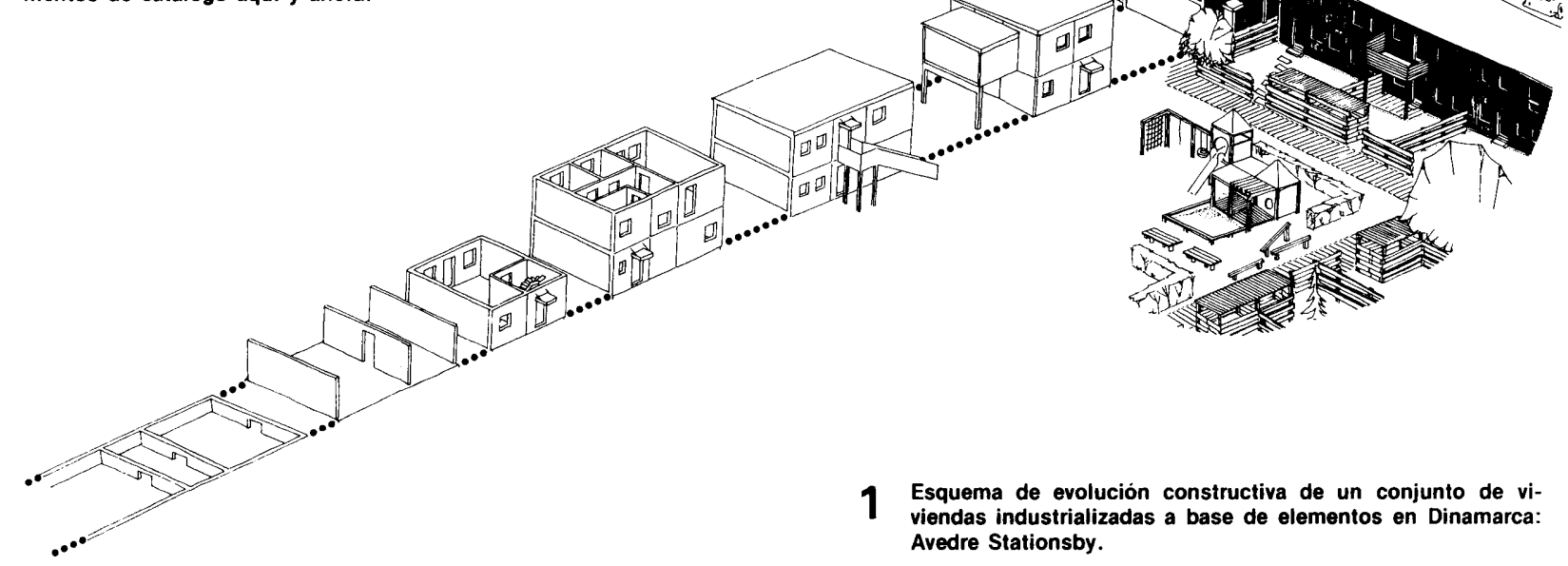




\section{Estereotomia de la vivienda en elementos funcionales}

El acercamiento al tema constructivo de la vivienda plantea, al menos, dos tipos de enfoques globales: la vivienda como un todo y la vivienda como un conjunto de partes o elementos funcionales de la misma. Ambos puntos de mira pueden responder a estrategias o filosofías distintas que intentaremos matizar. Trataremos de establecer una definición de los elementos funcionales que configuran una vivienda-tipo, intentando evaluar su posible repercusión económica (fig. 1).

Entenderemos como elemento funcional o unidad de proyecto "el grupo de variables que deben ser objeto de decisiones conjuntas de diseñon; estas unidades de proyecto tienen una correlación en la descomposición del edificio en elementos funcionales que pueden, o no, materializarse a base de componentes industrializados. Una escalera es una unidad de proyecto y puede ser también un componente industrializado. Lo que caracteriza a la similitud establecida entre componentes y unidades de proyecto es su valoración global en la que se incorporan materiales,

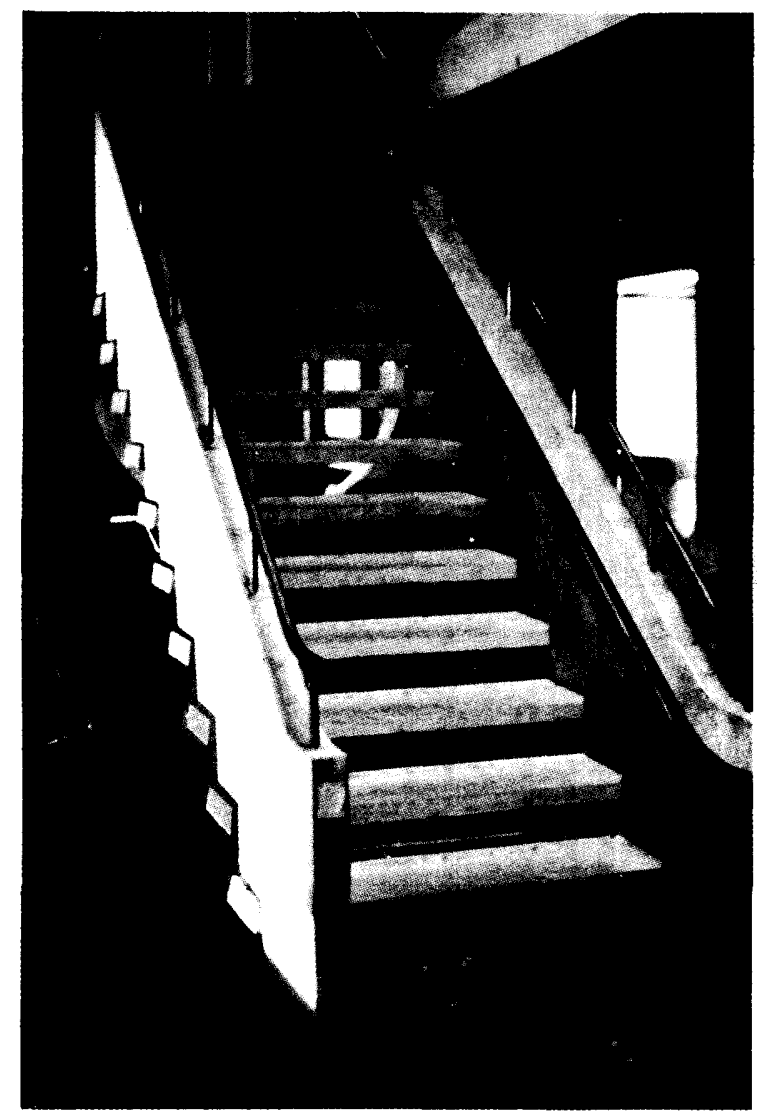

2 Ejemplo de elemento funcional traducido en componente industrializado. elementos, accesorios y tareas que conforman su función específica. En el ejemplo anterior, la escalera, incluirá la valoración total de todo aquello que permite desarrollar la función de acceso en condiciones de seguridad, iluminación, ambientación, etc. de modo que pueda conocerse la magnitud económica final de este componente en el conjunto del edificio (figura 2).

A fin de poder concretar desde una óptica práctica y cotidiana, hemos seleccionado tres "viviendas-tipo" que juzgamos como "representativas" de una amplia gama del mercado actual y que llamaremos viviendas tipo A, B y C $\left(^{*}\right)$.

Tipo A: Vivienda en "bloque lineal" de cuatro plantas, con dos viviendas por planta, superficie útil de $72 \mathrm{~m}^{2}$, con eje de simetria longitudinal, tres dormitorios, estar-comedor, baño y cocina (fig. 3).

Tipo B: Vivienda en un "bloque puntual", de ocho plantas, con cuatro viviendas por planta, superficie útil de $70 \mathrm{~m}^{2}$, dos ejes de simetria, tres dormitorios, estar-comedor, baño y cocina (fig. 4).

Tipo C: Vivienda unifamiliar aislada, cubierta a dos aguas, superficie construida $136 \mathrm{~m}^{2}$, tres dormitorios, estar-comedor, baño y cocina.

Hemos supuesto equipamientos y acabados de calidad idéntica o similar en los tres casos; los resultados porcentuales sobre el coste de construcción de los elementos funcionales para los tres tipos de viviendas estudiadas se recogen en la figura 5.

Hoy, el mercado europeo más dinámico de componentes, subsistemas, productos y elementos de catálogo, apunta hacia la meta de conseguir mediante una gama abierta de componentes industrializados, que abarcase las funciones:

- estructura $(8,0 \%$ valores medios del gráfico),

- elementos horizontales $(16,6 \%)$,

(*) Los datos que siguen son parte de los resultados de un trabajo realizado por el autor en colaboración con el Arqto. Salvador Pérez Arroyo sobre una muestra de 26.249 viviendas, realizadas en la pasada década en 20 provincias españolas, siguiendo básicamente el "Procedimiento A.R.C." puesto a punto por el Prof. Noël del C.S.T.B. 


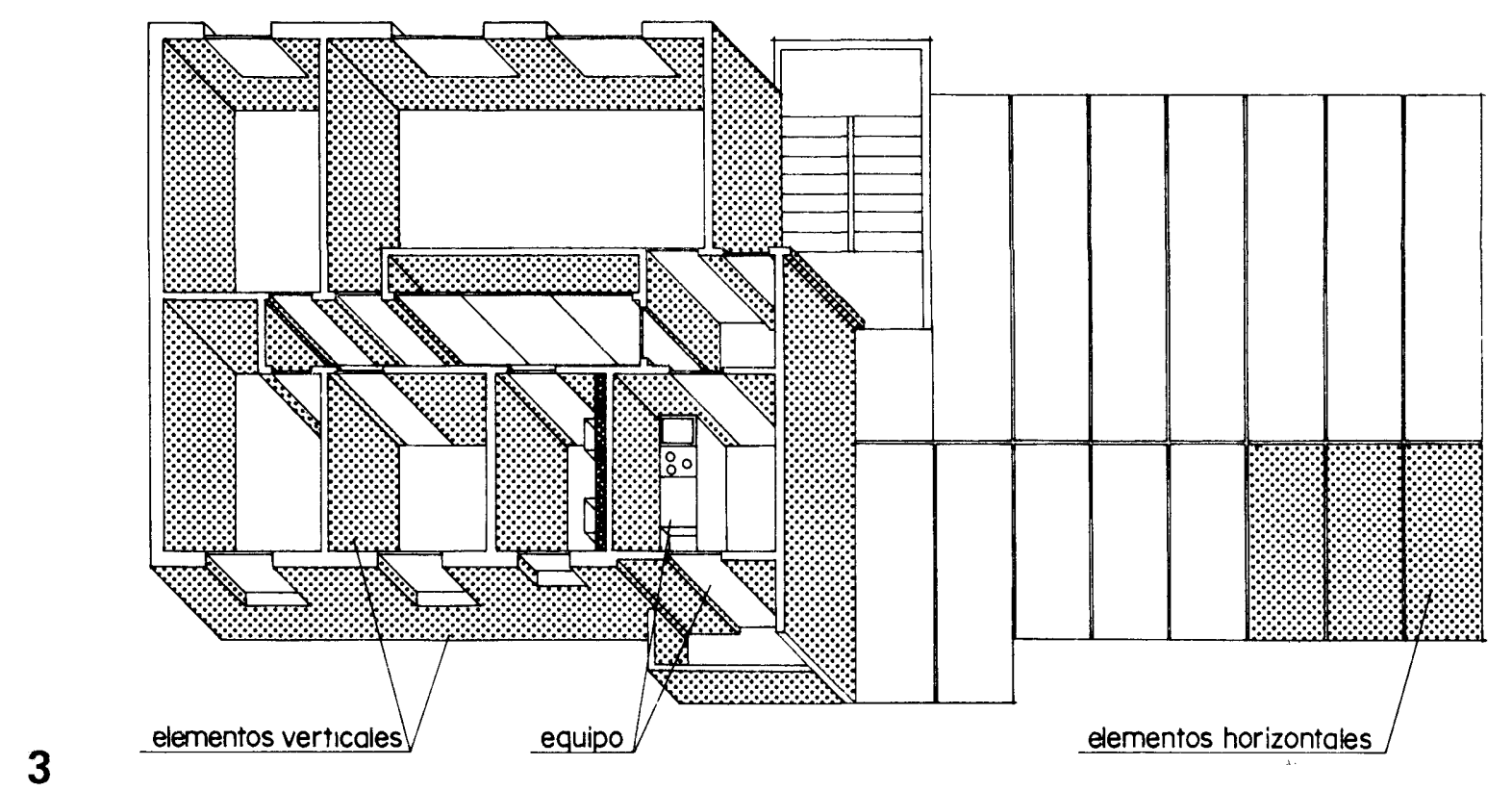

Bloque de tipo

lineal: elementos

horizontales, verticales

y equipo.

4

Bloque tipo puntual: elementos horizontales, verticales y equipo.

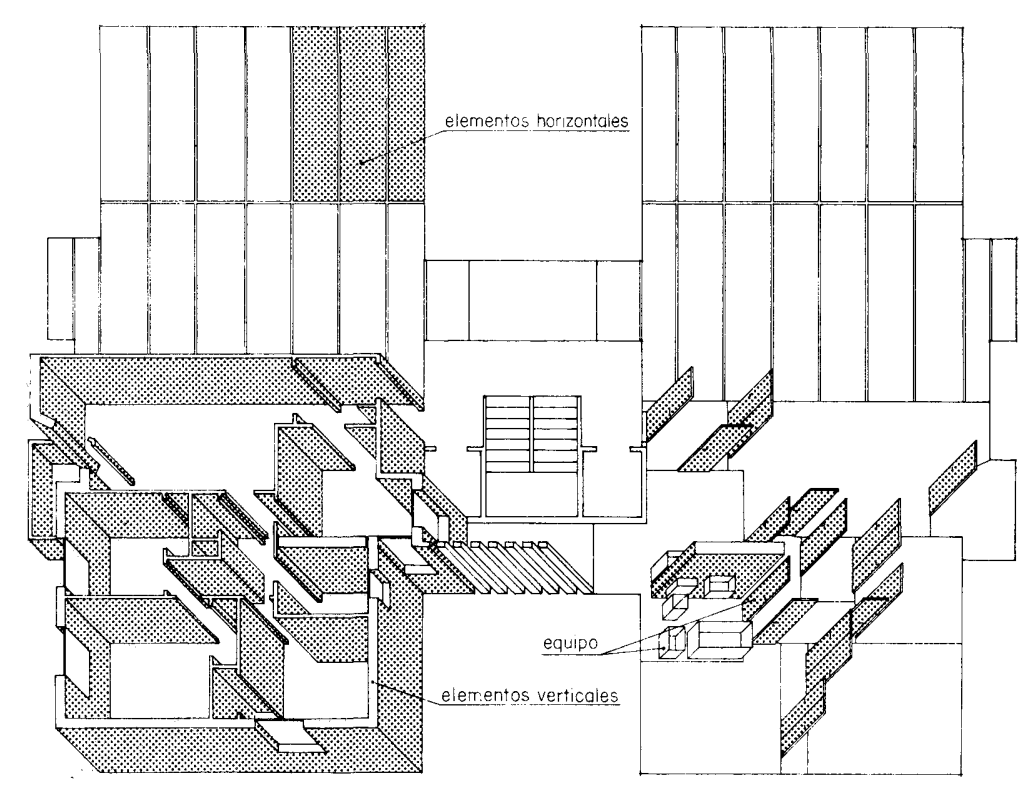

\footnotetext{
- elementos verticales interiores $(17,5)$,

- elementos verticales exteriores $(17,5 \%)$,

- equipo de la vivienda $(9,1 \%)$,

- cocina y baño $(9,3 \%)$,
}

repercusiones sobre el coste de construcción que podrian alcanzar, al menos teóricamente, un valor máximo de hasta el $70 \%$; valor sensiblemente superior al de cualquier sistema cerrado que dificilmente pasa del $40 \% \div 50 \%$.

\section{Componentes}

La ISO define los componentes de construcción como "Productos fabricados bajo la forma de unidades dispuestas para entrar a constituir partes de la construcción de una obra". Por su parte, el Grupo GERIA entiende los componentes como "partes invariables de edificios diferentes que deben responder a las exigencias funcionales, técnicas y económicas del cometido que se les encomienda, satisfaciendo por tanto condiciones de: compatilidad por su forma; de cocrdinación por sus dimensiones, y de diversidad combinatoria". 


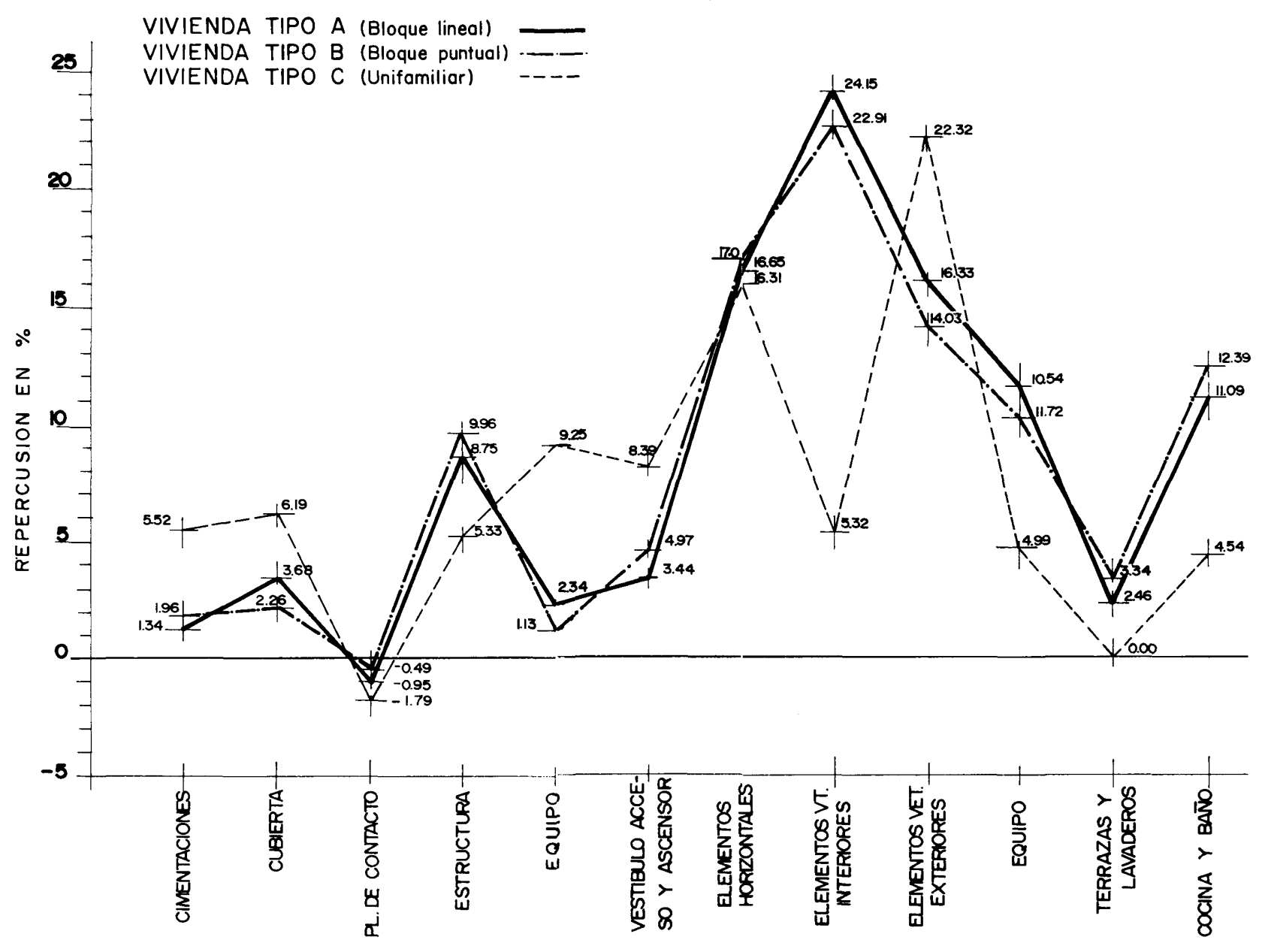

5

El interés del componente en el contexto de la industrialización abierta, está precisamente en que supone una renovación importante de formas, materiales, métodos de fabricación, resolución de juntas... renovación, que en muchos casos no es otra cosa que la actualización de soluciones ya utilizadas por Wachsmann, Gropius, Prouvé, Fuller... o de otras anónimas procedentes de la industria del aluminio, del plástico, de los laminados de madera... que cada día se acercan más al sector construcción, produciendo un tipo de industrialización que calificariamos de sutil infiltración.

No se trata de esperar a que la industria ponga a punto nuevos procesos de fabricación para aplicarlos en la edificación; los procesos existen y son tradicionales en la industria: la laminación de acero, aluminio, plástico, madera, materiales sintéticos..., la extrusión continua de perfiles de cualquier sección compleja, la conformación por plegado, mandrilado, soldadura por puntos..., la producción continua de elementos multicapas (sand- wich) por inyección de espuma entre láminas, prensado, rociado de revestimientos, inmersión... Son técnicas de plena vigencia en otros sectores que esperan fundamentalmente alcanzar la necesaria competitividad, que no es ajena a la racionalización del sector.

Una de las constantes comunes a estas tecnologías es la tendencia al ahorro de materias primas y semielaboradas, ahorro que se consigue mediante la transformación altamente mecanizada de materias primas y semiproductos en elementos complejos, por lo general, más ligeros que las soluciones homólogas tradicionales.

Obviamente, todo no se reduce a los problemas de producción. La estructura sectorial juega un importantísimo papel, por ello, quienes proponen sin más el lanzar elementos sobre el mercado y esperar a que se produzca la compatibilidad universal, de la que germinará la industrialización abierta, nos parece que mitifican un mundo que casi nada tiene de idílico. 
El componente no irrumpe milagrosamente en el mercado. Negamos por utópica la compatibilidad universal; pero los hechos demuestran el resurgir de una compatibilidad acotada, delimitada, que por supuesto, no es espontánea. La industrialización abierta es una gran idea en la que la implementación depende del Estado que no debe ni aplastar a los constructores, ni dejar perecer a las primeras empresas que lanzan componentes industriales. Este es el cuello de botella de la cuestión. La industrialización abierta, fruto lógico del mercado de libre competencia, parece incapaz de nacer sin el cuidado protector del Estado. En la correcta solución de esta aparente contradicción se esconde parte de la clave del éxito que no se prodiga.

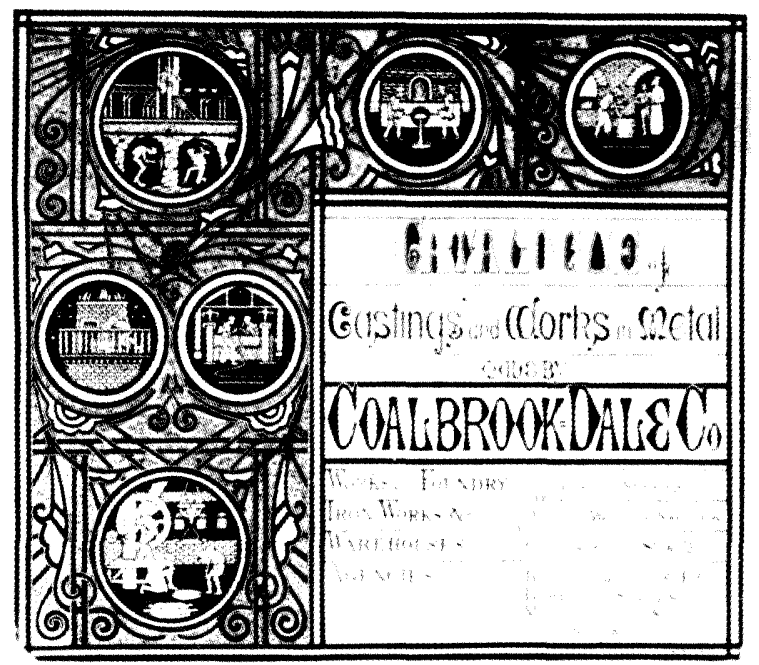

6 Portada del catálogo de 1860 de la empresa inglesa CoalbrookDale Co., la fundición que ejecutó en 1777 el maravilloso Ironbrige, que recientemente celebró su segundo centenario en uso.

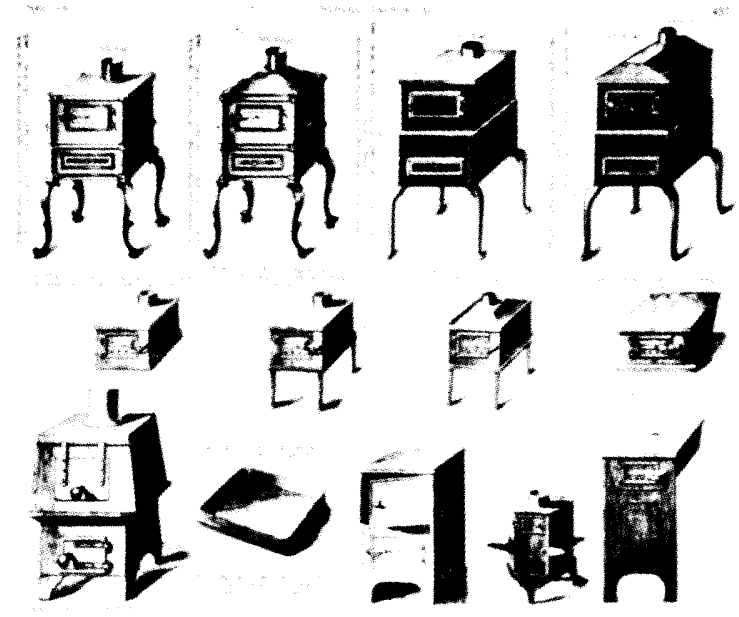

7 Una de las páginas, correspondiente a la sección de estufas domésticas, del catálogo de la Coalbrook.
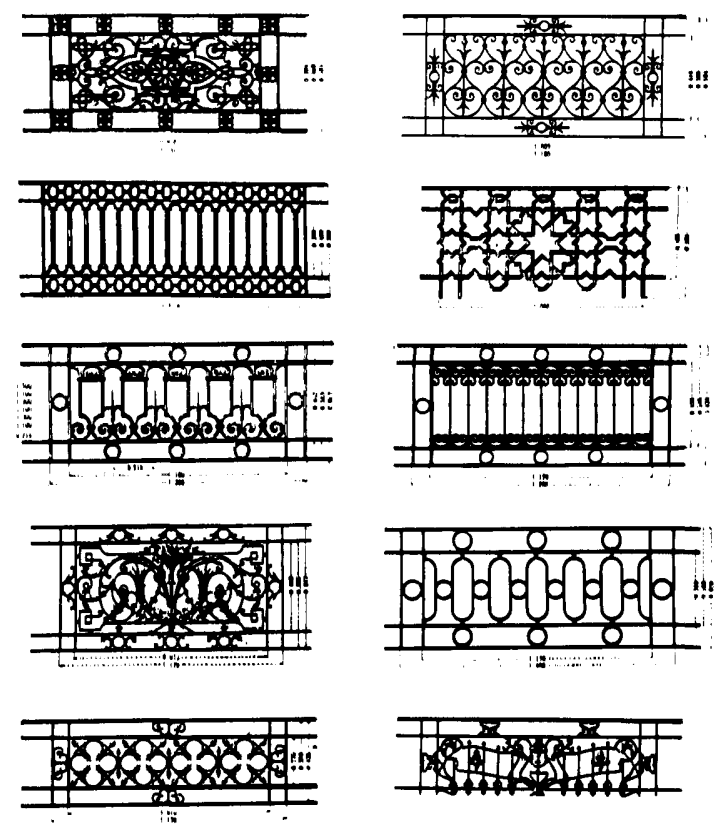

8 Una muestra de la extensa gama de balcones de fundición del catálogo Durenne. (Finales del XIX).

Siguiendo con la delimitación de lo que entendemos por componente, distinguiremos entre componentes especificos y componentes autónomos. Por específicos entenderemos los fabricados por un equipo determinado, de manera que puedan combinarse entre ellos de formas muy diferentes permitiendo arquitecturas muy variadas, pero siguiendo unas reglas bien definidas; componentes autónomos, por el contrario, son susceptibles de ser empleados fuera o dentro de un sistema en no importa qué tipo de uso atendiendo únicamente los condicionantes de su propia función.

Una vez aceptadas estas acotaciones, es fácil llegar a la receta:

\footnotetext{
Componentes específicos + componentes autónomos =

= sistema constructivo
}

Enunciaremos cuatro procesos o niveles de construcción a base de elementos y componentes industrializados:

Sistemas conocidos como cerrados en la década de lo sesenta

Los elementos se fabrican de acuerdo con las especificaciones internas del sistema; únicamente debe existir una compatibilidad entre proyecto y respuesta del sistema. 


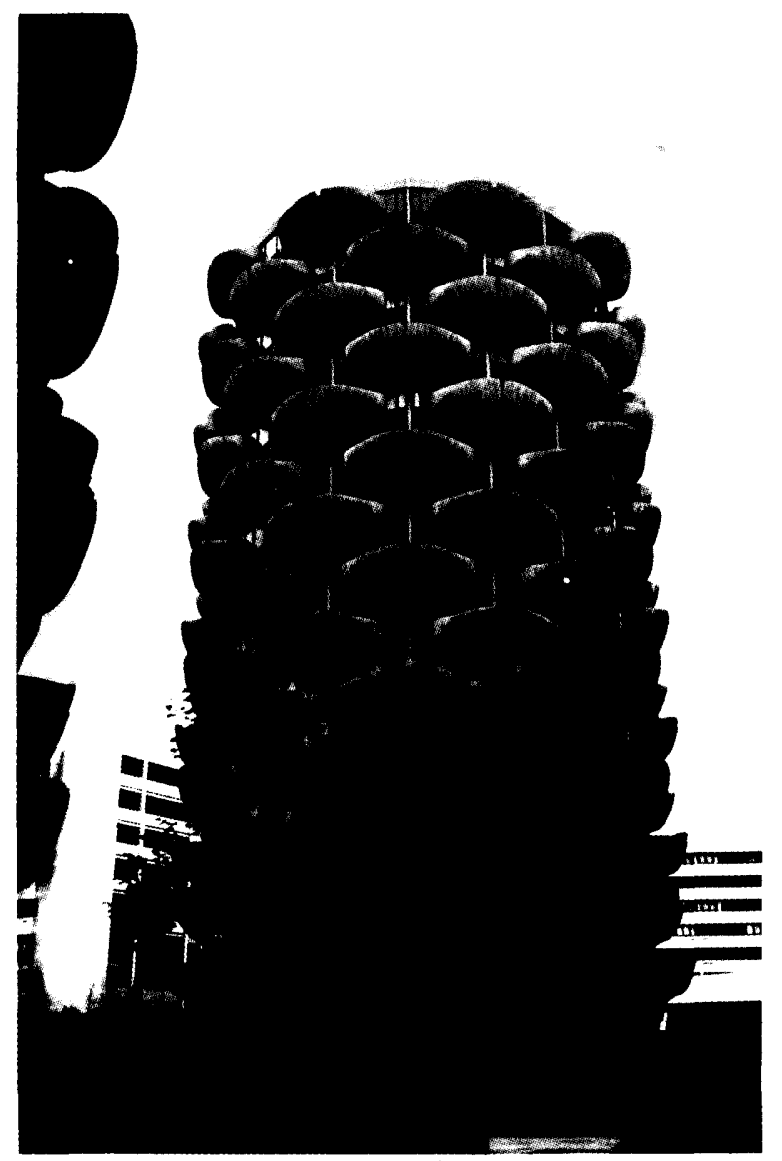

9 Una muestra de utilización simbólica de elementos de encargo de "hormigón arquitectónico" en un conjunto de Pantin (París).

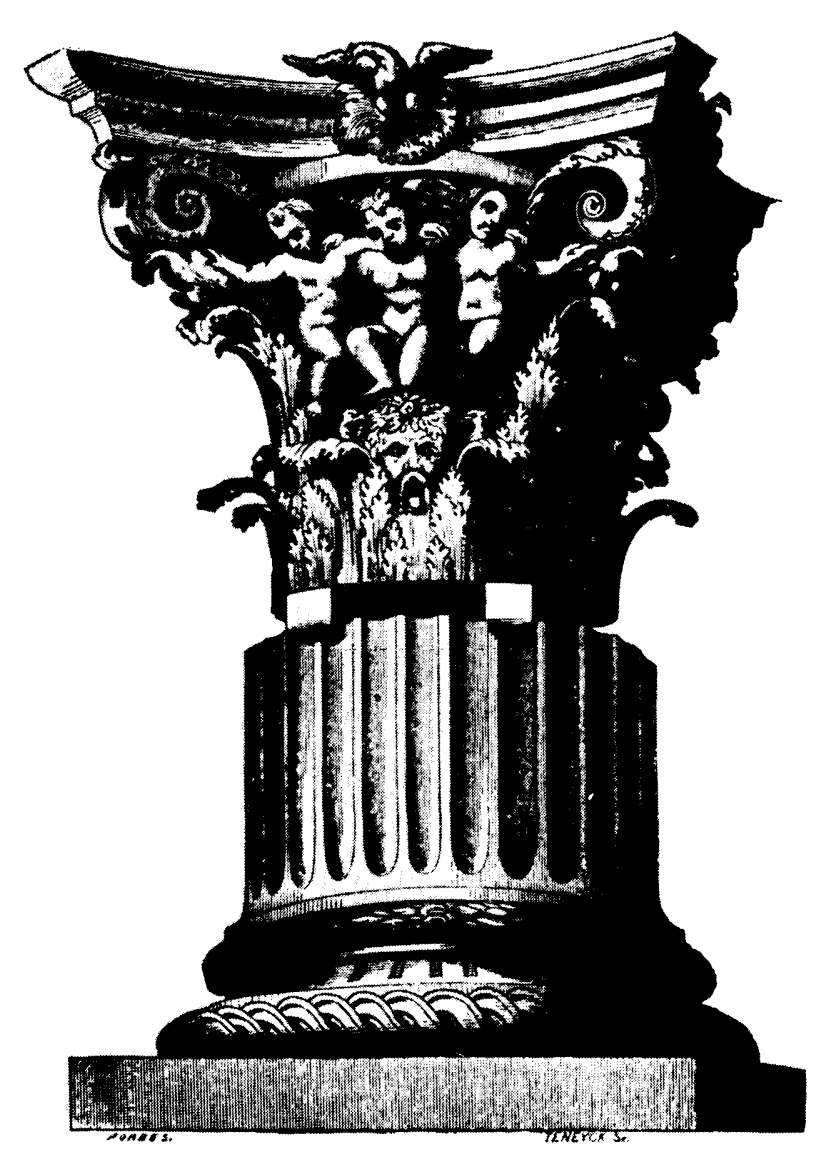

10 Base y capitel de columnas fundidas pertenecientes al catálogo de la casa francesa Denonvilliers de 1880 .

\section{Empleo parcial de componentes}

La gama de productos y prestaciones es más o menos fija admitiéndose cierta variedad dimensional.

\section{Sistemas tipo mecano}

Se trata en parte del antiguo sistema cerrado preparado para combinarse en múltiples soluciones atendidas por distintos productores concurrentes que respetan un mismo lenguaje combinatorio.

\section{Sistemas abiertos}

Están constituidos por elementos o componentes de distinta procedencia aptos para ser colocados en distintas obras, industrializadas o no, y contextos; sirviéndose de juntas "universales", gamas modulares, etc., principios que son difíciles de establecer con carácter general y que suponen un fuerte condicionante al diseñar con el sistema.

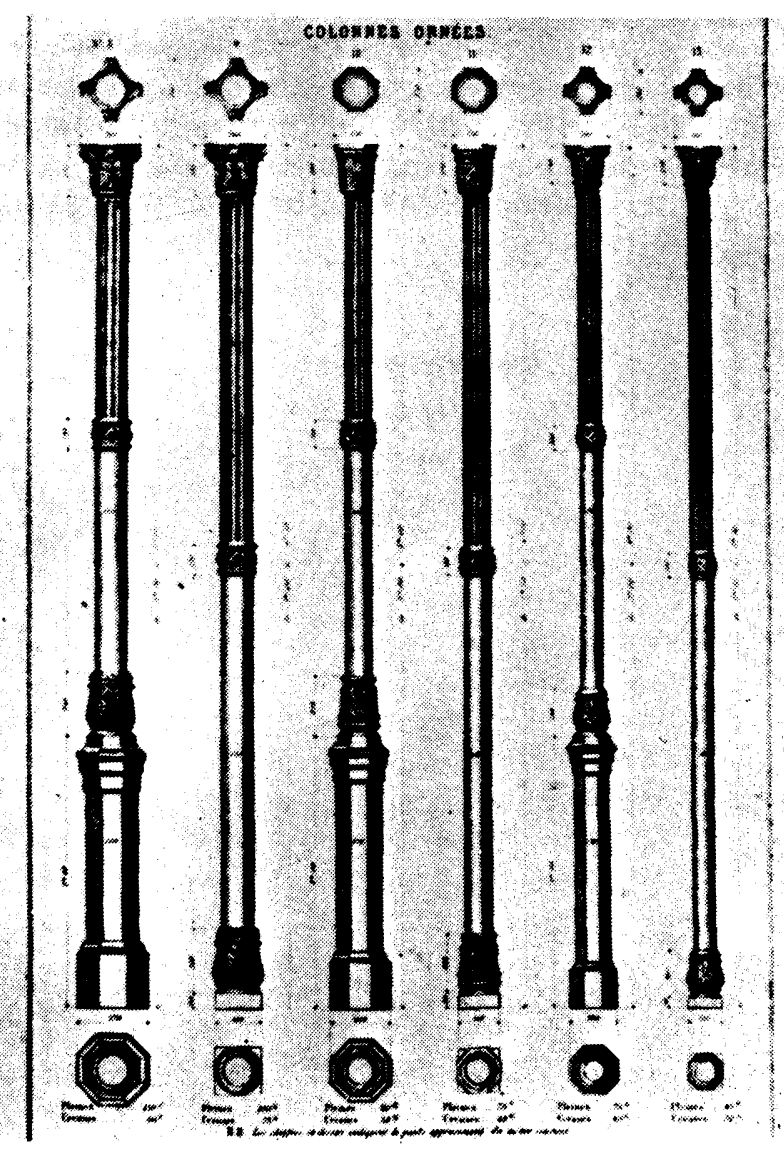
11 Catálogo de columnas de la casa Denonvilliers, fechado en
1880. 


\section{El origen de los catálogos de elementos}

Previamente a entrar en el tema de los catálogos de elementos constructivos, pensamos que puede ser interesante, por sus ricas consecuencias prácticas, el intentar un acercamiento a la andadura que hace algo más de un siglo recorrieron los productores de elementos de fundición.

Hemos de remontarnos a finales del XVII para encontrar en el "Cours d'architecture", de Charles Augustin Dabiler, las primeras referencias de objetos de fundición en un tratado de arquitectura. Años más tarde, la fundición tenía ya una presencia notable, en la construcción, en Francia e Inglaterra. Es en el período 1840-1880 -que $F$. Chaslin denomina "edad de oro" de los catálogos - cuando puede hablarse de una presencia cuantitativamente importante de los productos de acero y hierro fundido en el mercado de la construcción, apoyados en catálogos extraordinariamente ricos donde la suntuosidad de sus láminas primaba sobre la información que ofrecian respecto a dimensiones, caracteristicas, combinatoria, posibilidades y precios. (Figuras 6,7 y 8 ).

Nos parece interesante señalar que el catálogo de André de Val d'Osne (Francia), que se componia de 177 planchas en 1858, pasó a 367 en 1864 y llegó a 950 en 1878 . Trayectoria similar sigue otro de los catálogos más importantes de la época, el de A. Durenne, que pasó de 290 láminas en 1867 a 677 en 1872. El coste de estos

\section{LAVABO MURAL}

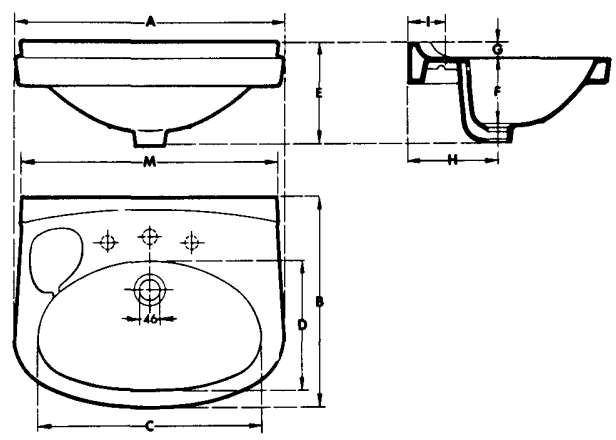

\begin{tabular}{l|l|l|l|l|l|l|l|l|l|l} 
& A & B & C & D & E & F & G & H & I & M \\
\hline
\end{tabular}

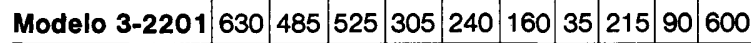
\begin{tabular}{l|l|l|l|l|l|l|l|l|l|l}
\hline Modelo 3-2202 & 560 & 440 & 465 & 280 & 220 & 145 & 30 & 200 & 75 & 535 \\
\hline
\end{tabular} \begin{tabular}{|l|l|l|l|l|l|l|l|l|l|l}
\hline Modelo 3-2203 & 510 & 410 & 450 & 250 & 210 & 135 & 30 & 200 & 75 & 490
\end{tabular}

12 Un ejemplo lipico de gama dimensional de componentes autonomos: aparatos sanitarios.
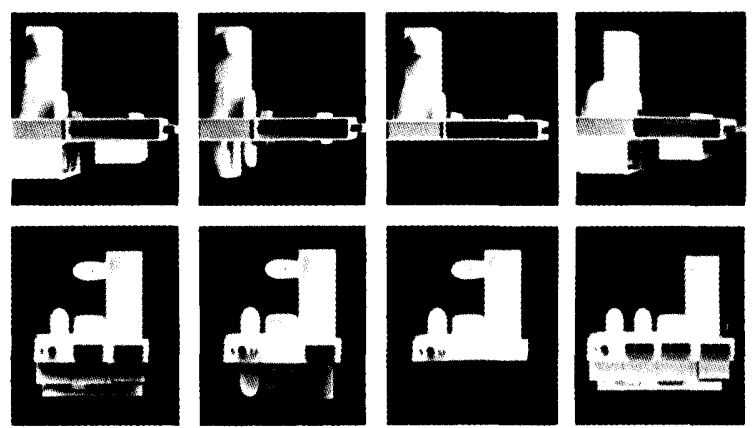

13 Gama parsi.

catálogos llegó a ser tan considerable que Barbezat \& Cía., los vendia a 30 Francos (1865) y la mencionada casa André, cuya tarifa de precios constaba de ¡300 páginas!, cobraba 40 francos por el catálogo, reembolsables caso de comprar más de $2.000 \mathrm{francos} /$ año.

El interés del tema, lógicamente, no está en el coste de los catálogos, sino en la problemática que se le plantea a la industria de la fundición: variedad versus economia. El tema se planteó un siglo más tarde, en idénticos términos, en el sector de prefabricación y más concretamente al llamado "hormigón arquitectónico". (Figura 9). ¿Cómo armonizar la proliferación de modelos distintos requeridos, con las inversiones en moldes?... La solución no llegó y los parques de los talleres se llenaron de montañas de moldes para chatarra. Guettier levanta acta de este hecho en 1844: "después de algunos años, la necesidad de moldes se ha desarrollado tanto que varios maestros fundidores han considerado conveniente el consagrar cuantiosas sumas a esta parte del material"; las industrias se arruinarán, predice, si ellas ceden a "las necesidades de cambio que se presentan hoy dian.

Algunas décadas después, coincidiendo con el declive de esta industria en Francia e Inglaterra, cuya inflexión puede fijarse en 1980, Guettier escribia: "En estos últimos años, la situación de los encargos no ha aumentado, los fabricantes han creado nuevos modelos, y por todas partes, los arquitectos han aumentado el uso del acero abandonado la fundición... Una parte de las fábricas productoras de fundición ornamental ha desaparecido y sólo queda un pequeño numero de establecimientos que se han fusionado. El precio de la fundición baja sin cesar al tiempo que los caprichos de los arquitectos 
aumentan; no es posible crear indefinidamente nuevos modelos sin ir a la ruina. Nos hemos parado y hacemos bien. Esto es angustiante...". La vigencia de esta cita centenaria nos parece maravillosamente profética; idénticos razonamientos hemos escuchado de productores de elementos prefabricados por encargo, después de haber intentado "defender" un catálogo que no fue posible.

Encontramos una diferencia, fundamental, en esta serie de paralelismos coincidentes entre prefabricación y fundición, que jugaba en detrimento de la difusión de esta última: la ornamentación. A la serie de problemas que dificultan la combinatoriedad entre elementos: dimensionales, estructurales... habia que añadir la de conseguir una compatibilidad ornamental. Habia que elegir entre los distintos órdenes, entre distintos estilos, entre las distintas modas: victoriana, flamboyante, arabesca, ... Por el contrario, el neoyorquino Bogardus se felicitaba de la happy adaptability de la fundición que permitia la ornamentación para todo tipo de gustos, y "que podia reproducir cualquier estilo arquitectónico». (Fig. 10).

Uno de los "componentes" de fundición que tomó carta de naturaleza fue la columna. Las más corrientes eran cilíndricas, de diámetros que oscilaban de 6 a $14 \mathrm{~cm}$ y alturas de $2,435 \mathrm{~m}$ a $3,896 \mathrm{~m}$ (múltiplos de la antigua pulgada). Posteriormente, en 1860, eran de catálogo columnas desde $1.50 \mathrm{~m}$ a $6,00 \mathrm{~m}$, y por encargo se fabricaban elementos para dos y tres plantas de altura. (Fig. 11).

\section{Los catálogos como camino hacia la industrialización abierta}

¿Estamos en el reinicio de la etapa de los catálogos? La idea del catálogo de componentes, indudablemente seductora, se presenta como una posible vía para cimentar un futuro de industrialización abierta. Esta, no ha de olvidarse, necesita de un determinado nivel de desarrollo de componentes. Nivel impreciso, pero indudablemente alto ya que según criterio de Prouvé, Francia aún no lo ha alcanzado, y en opinión tan autorizada como la de Colin Davidson: "La industrialización abierta, esa solución milagro que permite construir a base de componentes, aún no ha aparecido en América del Norte".
Hemos diferenciado anteriormente entre componentes especificos y componentes autónomos. En la materialización de sus respectivos catálogos encontraremos manifiestas diferencias entre ambas familias de componentes. Los "componentes autónomos" presentan un catálogo centrado en la descripción de caracteristicas del producto; tratan de sugerir empleos y utilizaciones sin carácter exhaustivo. Componentes autónomos típicos son los semiproductos, los cuales presentan una evolución clara hacia el "pret a poser", hacia el "bricolage" en la vivienda.

La placa cortable a medida, se llena de contenidos y prestaciones, se hace sandwich, barrera de vapor, paramento visto...; el perfil por extrusión gana en complejidad, se hace estanco, aloja conducciones, puede ser recuperable...; el rollo de material impermeabilizante de cubiertas aisla, impermeabiliza, se moldea en formas complejas, sella juntas... Estos semiproductos, componentes autónomos en un buen número de casos, que no aceptan el permanecer prisioneros de un mercado de "sistemas y procedimientos" trata de buscar otras formas de uso y aplicación, las propias de útiles adaptadores. Los catálogos de semiproductos se presentan como panoplia con vocación de abrirse al conjunto de los usuarios.

Típicos componentes autónomos, tradicionalmente catalogables, son la amplia gama de productos acabados implantados en el mercado, que constituyen las partes separables del edificio y/o vivienda: ascensores, radiadores, aparatos sanitarios, equipos de cocina, "shunts" (figuras 12 y 13). En general, no presentan problemas de compatibilidad, si se han previsto en proyecto, ya que suelen emplearse como útiles adaptadores o con la ayuda de otros aún más flexibles de tipo tradicional-artesanal ejecutados in situ. Para facilitar su empleo, existe una tendencia a la agrupación y clasificación nacional de estos catálogos en forma de volúmenes sistematizados.

Los catálogos de componentes especificos presentan un cometido bien distinto al de "sugeridor", que hemos dado como definitorio de los anteriores. Estos catálogos tienden a facilitar las ureglas de juego internas" mediante las cuales pueden utilizarse eficazmente los elementos constituyentes. Los catálogos de componentes 
especificos suelen presentar: gamas de elementos básicos, alfabeto (figs. 14 y 15); reglas o procedimientos de unión, sintaxls (fig. 16); ejemplos de uso mediante la aportación de esquemas o detalles neutros, que constituyen ejemplos de sintaxis regular, dejando ablerto un extenso campo de posibilidades para la sintaxis flgurada (siempre en el sentido gramatical) "que no es, como pudiera creerse, hija del capricho artificioson, pero que tampoco limita o cierra las posibilidades a "sentir un latido de alegría al encontrar un adjetivo acoplado con felicidad a un sustantivo, que nunca se vieron juntos" según frase de $C$. Pavese en "El Oficio de Poetan.

En algunos casos reales de la práctica constructiva, el catálogo de componentes específicos es extraordinariamente reducido pudiendo presentar un número de posibles soluciones muy amplias. Pensamos en el caso de estructuras tridimensionales
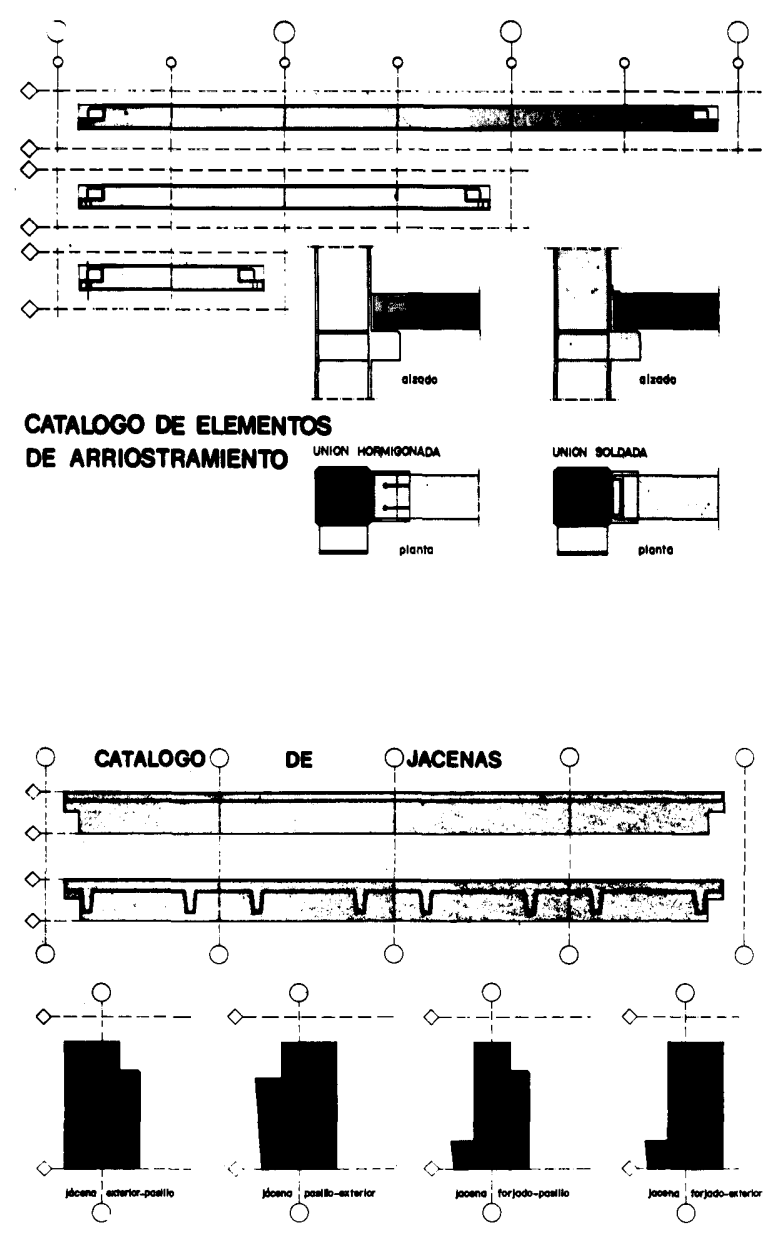

14-15 Alfabeto parcial de elementos estructurales de hormigón, ejemplo de componentes especificos del subsistema estructura porplo de
MODULACION HORIZONTAL $\$ 3 M \cdot 30 \mathrm{~cm}$

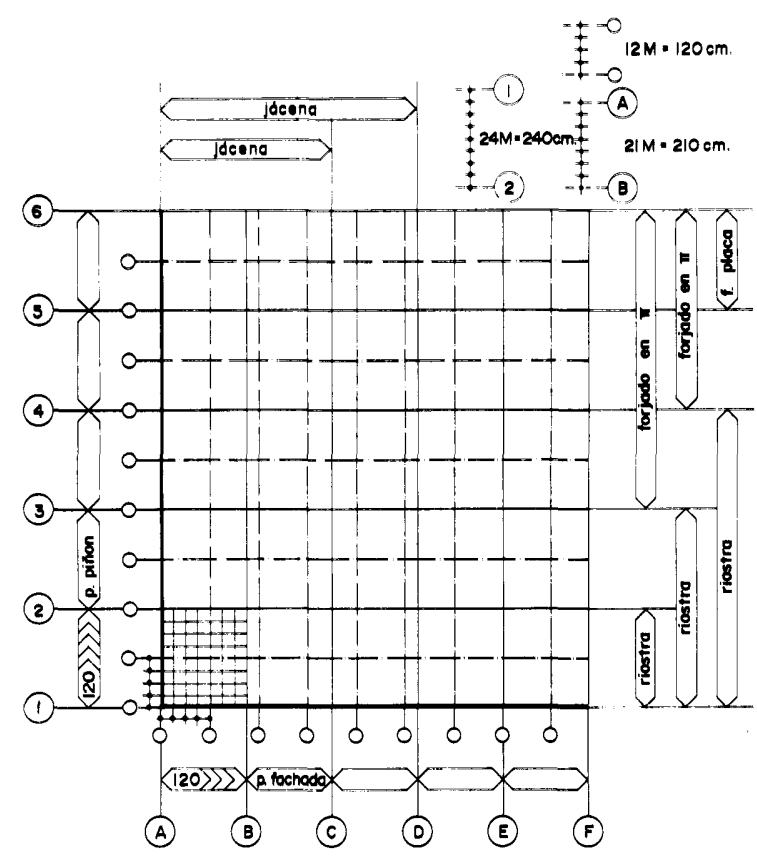

16

Sintaxis parcial (modulación horizontal) de los elementos de catálogo recogidos on las flguras anterlores.

de cubierta, en las que un tipo de nudo (MERO, Whachsman...) y unas pocas barras diferentes, generan multitud de formas y se adaptan a usos indistintos.

Dentro del simplismo que supone el tratar de reflejar en una ecuación la complejidad de un raciocinio, propondriamos como resumen:

Componentes lespecíficos y/o autónomos + + ejecución (racionalizada $y / 0$ tradicional) =

$=$ resultado construido

De la preponderancia de una u otra de las posibilidades de cada sumando tendremos el carácter industrializado o tradicional de la obra construida. Caso de que el carácter industrializado fuese preponderante, de la relación entre los tipos de componentes, deduciriamos el mayor o menor grado de abertura. Sistema cerrado clásico sería, según estos criterios, el constituido mayoritariamente por componentes específicos con muy poco uso de componentes autónomos y escasa ejecución de carácter tradicional-artesanal.

\section{¿Hacia un embrión de reglas de juego?}

No es difícil recopilar puntos de vista, describir realizaciones y reseñar formas de actuación de una práctica de la industrialización abierta, que en general, nos es 
ajena, pero ¿estamos en condiciones de abcrdar un camino propio? Pensamos que podía merecer la pena el intentar la búsqueda de unos planteamientos mínimos y elementales sobre los que cimentar un posible desarrollo racional de futuro.

Por razones obvias, lamentamos no contar con el papel de motor o de árbitro activo que en este sentido podía jugar la Administración. Papel claramente asumido y explicitado por la Administración francesa al presentar y aprobar en el Parlamento como programa de acción prioritaria: "Promover nuevas técnicas para una construcción de calidad". Otro caso de administración activa sería la danesa a la que nos referiremos más adelante.

Nuestros objetivos deberian ser $\tan$ modestos como realistas y habrian de aflorar y desarrollarse, en estrecha relación entre profesionales, constructores y el conjunto de productores de elementos, sin dejar de buscar el apoyo de los medios más dinámicos de la Administración, y por supuesto, teniendo muy en cuenta las distintas experiencias internacionales así como los resultados que de ellas se han derivado.

Estamos pensando en un embrión de reglas de juego que facilitase el ir introduciendo componentes en el edificio. Cuando son pocos los componentes que llegan a obra, los problemas se revuelven in situ. Cuando se quiere llegar a un 30, 40 o mayor tanto por ciento respecto del costo total a base de componentes, la necesidad de unas reglas globales resulta imprescindible.

Coincidimos plenamente con el Prof. Ciribini cuando afirma en el prólogo de nuestro libro "ALOJAMIENTO Y TECNOLOGIA: ¿INDUSTRIALIZACION ABIERTA? que: "Superada la utópica idea de la coordinación dimensional como instrumento resolutivo en sí de la industrialización, como si bastase normalizar una dimensión de referencia y algunas magnitudes derivadas para asegurar las condiciones de compatibilidad, intercambiabilidad, sustituibilidad entre componentes, hemos aprendido con el tiempo, que debe entenderse aquélla, al contrario, como una estructura compleja, que ha de traducirse paulatinamente, en hechos concretos". Unas ciertas normas o directrices de coordinación dimensional de carácter concreto, asumibles por la mayoría de los interesados, puede ser un importante soporte para el fomento de una amplia producción industrial de componentes.

Al igual que en otros paises, nos encontramos atrapados en un círculo vicioso; cada eslabón del proceso constructivo espera a algún otro. Arquitectos e ingenieros de diseño rehusan describir el empleo de componentes modulares y adaptan sus proyectos a los que pueden obtener en el mercado; los fabricantes, por su parte, retienen el iniciar la producción de componentes modulares hasta tener demanda potencial suficiente.

¿Es labor de las autoridades públicas el romper el círculo vicioso? En Dinamarca, se ha hecho a través de la legislación para la construcción, la cual incorpora un principio por el cual las viviendas, edificios de oficinas, escuelas, residencias, guarderias, etc., deben diseñarse de acuerdo con los principios modulares establecidos en las Normas Danesas, basadas en las dimensiones internacionales, el módulo básico $1 \mathrm{M}(10 \mathrm{~cm})$ y el módulo horizontal $3 \mathrm{M}(30 \mathrm{~cm})$. Los requisitos no están formulados como exigencia de que los componentes modulares de construcción sean los que tengan que usarse, sino que, únicamente se requiere un proyecto de diseño tal, que los componentes modulares de construcción puedan usarse en el proyecto sin que éste tenga que alterarse.

Como resultado de esta iniciativa, no sólo se ha roto el círculo vicioso, sino que además, un número considerable de componentes de dimensiones modulares se fabrican hoy como "productos de catálogo" para un mercado anónimo.

¿Hasta dónde deberian llegar nuestras reglas de juego o de compatibilización de elementos?

Nos inclinamos más por la resolución concreta de problemas cotidianos entre componentes reales, sin hipotecar el desarrollo futuro, que por un enfoque academicista, globalizador y teórico. Hoy, ante la atonia del presente, nos parece más oportuno desbloquear dificultades reales que auscultar un hipotético desarrollo futuro más global.

Entendemos, que cualquier intento de abordar el tema de unas posibles reglas de juego para facilitar la compatibilización 
de componentes, aqui y ahora, ha de pasar por:

A. - Un conocimiento actualizado y en profundidad de la realidad del mercado nacional de componentes, elementos, productos, subsistemas: capacidad de producción instalada; procesos de producción; problemas de compatibilización; posibilidad o no de modificar los parámetros dimensionales; características de los mercados... para lo que es premisa imprescindible que los productores sientan la necesidad de orquestar dichas reglas de juego.

B. - Asimilación crítica de las actuaciones a nivel internacional. Como más significativas apuntamos: la práctica "B.E.S." en Finlandia; la experiencia del grupo holandés "S.A.R." y su norma nacional NEN 2883; el desarrollo y aplicación de las Convenciones Generales francesas "A.C.C."; la rica experiencia danesa... Seria necesario afrontar esta etapa de valoración bajo un prisma de múltiples facetas: posibilidades de diseño; dificultades de producción; distorsiones de mercado; caracteristicas funcionales; repercusiones económicas...

C.-Realización de un plan de trabajos sobre la normativa vigente y el parque de viviendas de reciente construcción, de cara a analizar las posibles descomposiciones en elementos funcionales, y su ejecución a base de componentes, elementos, productos y subsistemas de mercado.
D. - Proyecto, ejecución, ensayo y valoración de realizaciones piloto a nivel de: componentes, elementos funcionales complejos y construcciones completas.

E.-Elaboración, como resultado directo de las etapas anteriores, de un conjunto de reglas mínimas consensuadas y asumibles por la mayoria de los productores, que sin hipotecar el futuro, dinamizasen de forma tangible la situación presente.

Si mediante un plan coordinado de trabajo (fig. 17), y el esfuerzo colectivo de los diferentes actores del hecho constructivo se lograse pasar del lenguaje monosilábico y balbuciente de nuestros catálogos actuales, a la articulación de frases y discursos complejos, el salto sería encomiable. Pero no nos engañemos, con realismo, no puede pensarse en el fruto sin haber plantado el embrión y sin tener presente las dificultades que pueden aparecer antes de que sea tiempo de cosecha.

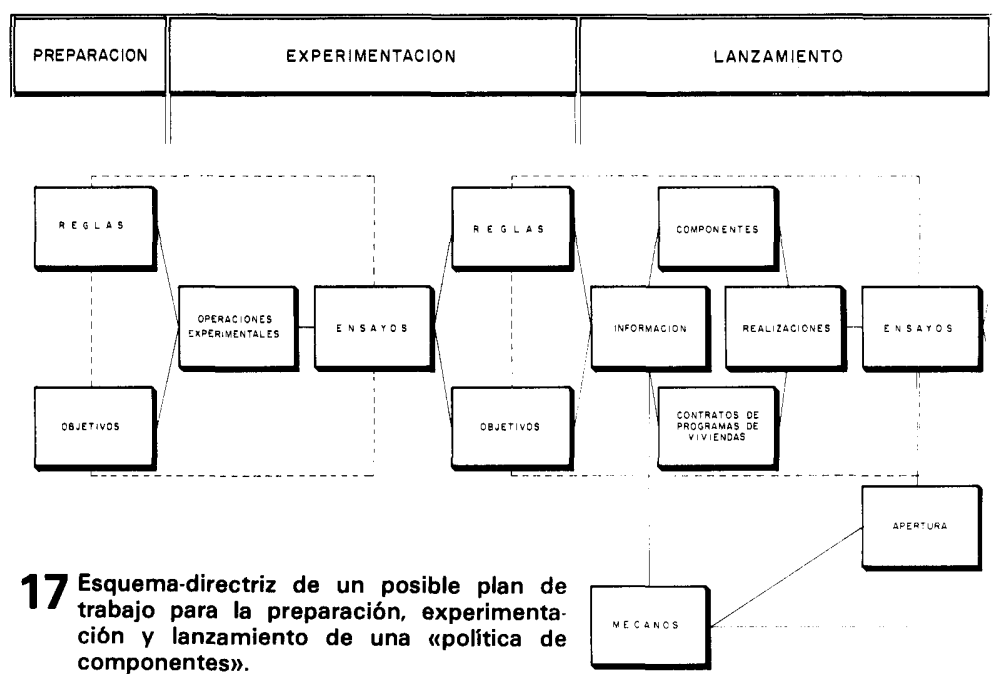

résumé

COMPOSANT ET ELEMENTS DE CATALOGUE: UNE VOIE DE RATIONALISATION DU PROCESSUS CONSTRUCTIF

Julián Salas, ingénieur industriel

L'auteur fait une analyse du concept de composant constructif, de sa répercussion économique, par une division de l'habitation en eléments fonctionnels, ainsi que des différents usages qu'on peut faire des différents usages qu'on peut
composants dans la construction.

La question des éléments de catalogue est posée par la recherche de leurs origines dans les catalogues d'éléments de fonte du XIXeme siecle établissant plus tard une division entre les éléments spécifiques division entre les élements spécifiques et les éléments autonomes. Finalement, conditions minimales pour aborder des regles de jeu autochtones qui faciliteraient composants et d'éléments de catalogue.

\section{summary}

COMPONENTS AND CATALOG ELE. MENTS: A WAY TO RATIONALIZE THE BUILDING PROCESS

Julián Salas, Industrial Engineer

An analysis is made of the concept of building component, its economic repercussions, by means of a division of the dwelling into functional elements, as well as the different uses that can be made of as the different uses that can
the components in building.

The subject of the catalog elements is approached by searching for its origins in the 19th century catalogs of cast iron elements and later. by establishing a division between specific and autonomous elements. Finally, the study presents what could be minimum conditions in order to set up autochthonous game rules which would facilitate the use of components and catalog elements here and now.

\section{zusammenfassung}

KATALOGBESTANDTEILE UND -ELEMENTE: EIN WEG ZUR RATIONALISIERUNG DES BAUPROZESSES

Julián Salas, Ingenieurs

Es werden der Begriff der Baukomponente, deren Auswirkung in wirtschaflicher Hin deren Auswirkung in wirtschaflicher Hinmöglichkeiten der Bestandteile durch eine Aufteilung der wohnung in Funktionselemente geprüft

Das Thema der Katalogelement wird durch die Suche ihres Ursprungs in Katalogen von Gussteilen aus dem XIX Jhdt. aufgeworfen. Später unterscheidet man zwischen spezifischen und autonomen Elementen. Abschliessend wird darauf hingewiesen, welche Spielregeln zur Erleichterung der Venwendung der Katalogbestandteile und - elemente unter Mindestbedingungen hier und jetzt festgesetz werden könnten. 